Hospital Practice

\title{
How do we know who has had deep vein thrombosis?
}

\author{
David A. Sandler and J.R.A. Mitchell \\ Department of Medicine, University Hospital, Queen's Medical Centre, Nottingham NG7 2UH, UK.
}

\begin{abstract}
Summary: An attempt was made to identify all patients diagnosed as having deep vein thrombosis (DVT) in a large teaching hospital during one year. A review of the radiology records showed that 124 $(81 \%)$ of the 154 venograms performed were for suspected DVT, and that the diagnosis was confirmed in 57 (46\%). During the same period, the Hospital Activity Analysis (HAA) records revealed 174 episodes classified as 'phlebitis or thrombophlebitis' in 162 patients. Only 37 of these episodes (21\%) had been confirmed by X-ray venography and at least 29 patients were incorrectly classified by HAA. 'Medical' patients accounted for $54 \%$ of the episodes of DVT, yet in only $25 \%$ of these was the diagnosis confirmed by venography, while 'surgical' DVTs were less frequent but were more often confirmed by venography.
\end{abstract}

\section{Introduction}

The only certain way to diagnose deep vein thrombosis (DVT) is by venography, ${ }^{1}$ and this test will confirm the suspected diagnosis in only $50 \%$ of patients investigated. ${ }^{2,3}$ We therefore undertook a retrospective study to: (i) determine the use currently being made of X-ray venography in a teaching hospital; (ii) see how well this correlated with the ultimate diagnosis of DVT made on patients discharged from the hospital; and (iii) note any differences in the use of venography between specialties.

\section{Methods}

University Hospital, Nottingham is a 1400-bed teaching hospital sharing with a similar hospital the care of a catchment area of approximately 650,000 residents. It accepts referrals from a wide area; either from general practitioners or through the accident and emergency department, which is the only one for the whole conurbation. The year of 1985 was chosen for the study because it was the most recent year for which complete records were available. The results of all venograms performed by the X-ray department during that year were scrutinized. Details of the patient, the indication for venography and the result of venogram were recorded.

For the same period, the Hospital Inpatient Activity Analysis (HAA) reports were scrutinized. The HAA

Correspondence: D.A. Sandler, M.D., M.R.C.P.

Accepted: 28 September 1988 clerks classify the diagnoses of all patients discharged from the hospital and this is usually done by reference to the discharge summary. The condition that was the main reason for admission is recorded as the 'primary' diagnosis and other associated conditions are labelled as 'secondary' diagnoses. Note was taken of all patients who had a primary or secondary diagnosis of phlebitis or thrombophlebitis of the superficial vessels of the lower limb (coded 451.0); deep vessels of lower limb (451.1); unspecified site on lower limbs (451.2) and unspecified sites (451.9). For each patient admission identified, note was taken of whether pulmonary embolism also appeared in the classification as either the primary or a secondary diagnosis.

Using this approach, patients could then be categorized into three groups:

1. Those who were identified as having DVT in both the venogram and the HAA surveys;

2. Those identified only in the venogram survey (i.e. patients having a positive venogram, confirming a DVT, but not being identified by the HAA coding);

3. Those identified only in the HAA survey (i.e. patients not having a venogram).

A potential fourth group (that is, patients with a clinical diagnosis of DVT, and without a venogram, who were missed by HAA), could not be quantified using these methods.

The reasons for any discrepancy between these two methods of identification were sought by review of the case notes. Patients were arbitrarily allocated to the following specialties: Medicine, Surgery, Orthopaedics, Gynaecology, Geriatrics and 'Others'.

(C) The Fellowship of Postgraduate Medicine, 1989 


\section{Results}

During 1985, 154 venograms were performed on 145 patients. Table I shows that 124 of these venograms $(81 \%)$, in 115 patients, were for suspected DVT. This includes 3 patients who had two venograms on different occasions; 3 patients who had bilateral venography (each was counted as two venograms); and 1 patient who had bilateral venography on 2 occasions (four venograms). Fifty-seven (46\%) of the 124 venograms for suspected DVT were positive. Venography was refused on two occasions by one patient, and abandoned on two occasions because of technical problems in a further patient. Of the 124 venograms for suspected deep-vein thrombosis, the right leg was examined on 62 occasions ( 29 positive) and the left leg on 57 occasions (27 positive). Three arms underwent venography (1 positive) and the limb

Table I Details of all venograms performed during study year

\begin{tabular}{lc}
\hline Total venograms & 154 \\
\hline Suspected DVT & 124 \\
$\quad$ Lower limb & 119 \\
Upper limb & 3 \\
Unknown site & 2 \\
Before varicose vein surgery & 13 \\
During arterial surgery & 13 \\
For arterio-venous malformation & 1 \\
Reason unknown & 3 \\
\hline
\end{tabular}

investigated on 2 occasions (both negative) could not be determined.

The HAA survey showed that 174 admissions of 162 patients during 1985 were classified as phlebitis or thrombophlebitis. Ten patients had 2 admissions during the year and 1 patient was admitted 3 times. Table II illustrates the numbers of admissions for each of the HAA classifications of phlebitis or thrombophlebitis, and shows that only $37(21 \%)$ of the 174 diagnoses were confirmed by venography. Of these 174 admissions, pulmonary embolism was the primary diagnosis on 14 occasions (8\%) and was listed as a secondary diagnosis in $38(22 \%)$. Of these 38 secondary diagnoses, only 5 had had a venogram performed; DVT was thus diagnosed without objective confirmation on all 14 admissions with the primary diagnosis of pulmonary embolism and in 33 of the 38 admissions in which it was a secondary diagnosis.

When comparing those patients identified by the venogram survey to those identified during the HAA survey, it was found that of the 57 positive venograms performed for suspected DVT, only $30(56 \%)$ of these patients were classified by HAA as having phlebitis or thrombophlebitis as a primary or secondary diagnosis. Conversely, 6 of the 67 patients with a negative venogram $(9 \%)$ were later classified by HAA as having phlebitis or thrombophlebitis. Of these six patients, three had been classified by HAA as 'phlebitis or thrombophlebitis of unspecified site, lower limb' and the discharge summary indicated that a clinical diagnosis of 'superficial thrombophlebitis' had been made. However, a further patient who had been similarly

Table II Relationship between HAA diagnosis and venography

\begin{tabular}{|c|c|c|c|c|c|c|}
\hline$H A A$ code & Total & $\begin{array}{l}\text { ory } \\
\text { Venis } \\
\text { Confirmed }\end{array}$ & Total & $\begin{array}{l}\text { lary } \\
\text { osis } \\
\text { Venogram } \\
\text { confirmed }\end{array}$ & Total & $\begin{array}{l}\text { Venogram } \\
\text { confirmed }\end{array}$ \\
\hline \multicolumn{7}{|l|}{$\begin{array}{l}\text { Phlebitis or thrombo- } \\
\text { phlebitis of: }\end{array}$} \\
\hline $\begin{array}{l}\text { Superficial vessels, } \\
\text { lower extremity } \\
\text { (HAA code } 451.0 \text { ) }\end{array}$ & 3 & 2 & 1 & 0 & 4 & 2 \\
\hline $\begin{array}{l}\text { Deep vessels, } \\
\text { lower extremity } \\
\text { (HAA code } 451.1 \text { ) }\end{array}$ & 84 & 19 & 76 & 13 & 160 & 32 \\
\hline $\begin{array}{l}\text { Unspecified site, } \\
\text { lower extremity } \\
\text { (HAA code } 451.2 \text { ) }\end{array}$ & 3 & 0 & 4 & 2 & 7 & 2 \\
\hline $\begin{array}{l}\text { Unspecified site } \\
\text { (HAA code 451.9) }\end{array}$ & 0 & 0 & 3 & 1 & 3 & 1 \\
\hline All admissions & 90 & 21 & 84 & 16 & 174 & 37 \\
\hline
\end{tabular}


classified by HAA had a clear statement in the discharge summary that 'venograms were negative in both legs'. The other two patients were both classified by HAA as having 'phlebitis or thrombophlebitis of deep vessels, lower limbs': the first was admitted for varicose vein surgery which led to the venogram and the second patient's summary clearly stated 'suspected DVT not confirmed by venography'. The HAA coding was clearly incorrect in the latter two examples.

Twenty-seven patients with positive venograms were not classified by HAA as having had phlebitis or thrombophlebitis. One patient had been an outpatient throughout and therefore had not received HAA classification; two sets of case-notes could not be found; and the discharge summary relating to the admission of a further ten patients could not be located. However, of the remaining 14 patients in whom a discharge summary was found, DVT was clearly mentioned as a diagnosis in 11, but had not been so classified by the HAA.

For subsequent analysis, the 174 episodes of DVT identified during the HAA part of the survey, and the further 27 admissions found by virtue of a positive venogram, were considered together but the 4 patients with the HAA classification of superficial thrombophlebitis were excluded, as were 19 patients whose case-notes could not be found (17 from the HAA group and 2 from the venogram group). The remaining 178 episodes of DVT were categorized by specialty and Table III shows this division and the proportion of diagnoses confirmed by venography for each. The 7 patients in the 'Other' category consisted of 3 dermatology patients, 2 from neurosurgery and 1 each from rheumatology and neurology. Ninety-six (54\%) of the episodes of DVT were in 'medical' patients and a further $19(11 \%)$ were 'geriatric' patients. The general surgeons diagnosed DVT in 27 patients $(15 \%$ of all episodes) and a similar proportion were from orthopaedic surgical wards $(13 \%)$. It can be seen that gynaecologists and orthopaedic surgeons used venography in a higher proportion of their patients with suspected DVT (100\% and $58 \%$ respectively) than did

Table III Diagnosis of DVT with and without venography per specialty

\begin{tabular}{lcc}
\hline Specialty & $\begin{array}{c}\text { Number } \\
\text { with DVT }\end{array}$ & $\begin{array}{c}\text { Number (\% of specialty) } \\
\text { confirmed by venogram }\end{array}$ \\
\hline All patients & 178 & $56(31 \%)$ \\
Medicine & 96 & $24(25 \%)$ \\
Surgery & 27 & $8(30 \%)$ \\
Geriatrics & 19 & $4(21 \%)$ \\
Orthopaedics & 24 & $14(58 \%)$ \\
Gynaecology & 5 & $5(100 \%)$ \\
Others & 7 & $1(14 \%)$ \\
\hline
\end{tabular}

the other specialties, but that the numbers of DVT episodes which they dealt with only formed $16 \%$ of the total of 178 DVTs.

\section{Discussion}

This survey has shown that the diagnosis of DVT is made in far more patients than undergo X-ray venography, despite the fact that clinical examination is reliable in only about $50 \%$ of patients presenting with a suspected DVT. ${ }^{2,3}$ The HAA system classified phlebitis or thrombophlebitis in a total of 174 admissions, yet of these 174 admissions, only $37(21 \%)$ had a diagnostic venogram. This may be because the clinicians have already decided that the risks of anticoagulating a suspected DVT patient unnecessarily are outweighed by the risks of withholding treatment while venography is being arranged and performed. However, venography can still be performed on a patient who has already commenced heparin and this can quickly be discontinued if the test is normal. However, DVT is so common in some groups of high risk patients (at least $50 \%$ in patients with femoral neck fractures ${ }^{4}$ ) that if the clinicians requested venograms on all their 'suspected DVT cases' they would overwhelm their radiology colleagues and prevent other important tests from being done. Our study has shown that the vast majority $(81 \%)$ of venograms in this hospital were for suspected DVT. The finding that only $46 \%$ were positive would support other studies reporting similar rates of proven DVT in patients suspected of having this condition ${ }^{2,5}$ and if resources were adequate, would support a move towards the confirmation of DVT before subjecting patients to anticoagulant therapy.

Interestingly, only 3 patients underwent bilateral venography. We are therefore unable to comment on the frequency of clinically significant thromboses in the asymptomatic legs of patients investigated merely with unilateral venography. However, most authorities would probably agree that if DVT was suspected, especially if the patient presents with pulmonary embolism, phlebography should always be bilateral because of the frequency of clinically important DVT on the asymptomatic side. Although the present study was not designed to investigate the incidence and diagnosis of pulmonary embolism, the HAA survey demonstrated the co-existence of this with deep vein thrombosis in 52 of the 174 episodes studied $(30 \%)$. Of the patients classified as having pulmonary embolism, only 5 of $52(10 \%)$ underwent venography, even though the pulmonary embolism was classified as the 'secondary' diagnosis to DVT in $38(73 \%)$. If the diagnosis of DVT was confirmed in so few patients, one can only speculate as to how reliable the diagnosis of pulmonary embolism was in patients 
so classified. The 'surgical' specialties used venography in a higher proportion of their patients; patients with a 'medical' DVT had the diagnosis confirmed in only $25 \%$ of cases, a similar proportion to that found by Ramsay, ${ }^{6}$ yet 'medical' DVT accounted for $54 \%$ of all the DVTs diagnosed.

Many epidemiological studies rely on the accuracy of hospital records for information. It is known that the reporting of causes of death can be very unreliable, ${ }^{7}$ and this study repeats earlier questions which have been asked about the validity of information provided by the HAA services. ${ }^{8}$ Our survey has confirmed that there have been inaccuracies in classification such that if this information were to be used to plan future resource allocation, false estimates of the frequency of an important condition such as DVT in the hospital population would be provided. Three patients were incorrectly classified by HAA as having phlebitis or superficial thrombophlebitis when they clearly had neither, as recorded in their discharge summaries; and 26 inpatients who had a positive

\section{References}

1. Bettman, M.A., Robbins, A., Braun, S.D., Wetzner, S., Dunnick, N.R. \& Finkelstein, J. Contrast venography of the leg: diagnostic efficacy, tolerance, and complication rates with ionic and non-ionic contrast media. Radiology 1987, 165: 113-116.

2. Salzman, E.W. Venous thrombosis made easy. $N$ Engl $J$ Med 1986, 314: 847-848.

3. Whitehouse, G. Radiological diagnosis of deep vein thrombosis. Br Med J 1987, 295: 801-802.

4. Hirsh, J. Prophylaxis of venous thromboembolism. Mod Concept Cardiovasc Dis 1984, 53: 25-29. venogram for DVT were not classified as such by HAA - yet in at least $11(41 \%)$ of these patients the discharge summary, on which the HAA clerks are intended to base their classification, had clearly indicated that DVT was one of the diagnoses.

In conclusion, although the majority of venograms are performed for suspected DVT, most patients with 'DVT' diagnosed at this hospital did not undergo $\mathrm{X}$-ray venography, even though this is the most accurate diagnostic test. Furthermore, if anyone was trying to quantify the frequency of 'DVT' at this hospital, a study of HAA records, which would appear to be the only practical method of obtaining such information, cannot be relied upon to give accurate figures and underestimates the extent of the problem.

\section{Acknowledgements}

We would like to thank Dr E.J. Roebuck and colleagues in the X-ray department and Mr R. Nutt of Medical Records for access to the information necessary for the survey.

5. Charig, M.J. \& Fletcher, E.W.L. Emergency phlebography service: is it worthwhile? Br Med J 1987, 295: 474.

6. Ramsay, L.E. Impact of venography on the diagnosis and management of deep-vein thrombosis. Br Med J 1983, 286: 698-699.

7. Kircher, T., Nelson, J. \& Burdo, H. The autopsy as a measure of the accuracy of the death certificate. $N$ Engl J Med 1985, 313: $1263-1269$.

8. Martini, C.J.M., Hughes, A.O. \& Patton, V.A. A study of the validity of the Hospital Activities Analysis information. Br J Prev Soc Med 1976, 30: 180-186. 